Review Article

\title{
The Link between Circadian Clock Genes and Autophagy in Chronic Obstructive Pulmonary Disease
}

\author{
Yuedi Hu, ${ }^{1}$ Tiantian He, ${ }^{1}$ Jie Zhu $\mathbb{D}^{1,2,3}$ Xiaole Wang ${ }^{1},{ }^{3}$ Jiabing Tong, ${ }^{3,4}$ Zegeng Li, ${ }^{3,4}$ \\ and Jingcheng Dong $\mathbb{1}^{2}$ \\ ${ }^{1}$ College of Integrated Chinese and Western Medicine, Anhui University of Chinese Medicine, No. 1, Qianjiang Road, Hefei City, \\ Anhui Province, China \\ ${ }^{2}$ Institutes of Integrative Medicine, Fudan University, Shanghai, China \\ ${ }^{3}$ Institute of Traditional Chinese Medicine Prevention and Control on Respiratory Disease, Anhui Academy of Chinese Medicine, \\ No. 117, Meishan Road, Hefei City, Anhui Province, China \\ ${ }^{4}$ Department of Respiratory Medicine, First Affiliated Hospital of Anhui University of Traditional Chinese Medicine, Meishan Road, \\ Hefei City, Anhui Province, China
}

Correspondence should be addressed to Jie Zhu; janezhutcm@foxmail.com and Jingcheng Dong; jcdong2004@126.com

Received 8 April 2021; Accepted 8 October 2021; Published 25 October 2021

Academic Editor: Young-Su Yi

Copyright (C) 2021 Yuedi Hu et al. This is an open access article distributed under the Creative Commons Attribution License, which permits unrestricted use, distribution, and reproduction in any medium, provided the original work is properly cited.

\begin{abstract}
Chronic obstructive pulmonary disease (COPD), a progressive respiratory disease, is characterized by the alveolar epithelium injury and persistent airway inflammation. It is documented that oscillation and dysregulated expression of circadian clock genes, like Bmal1, Per1, and Per2, involved in COPD pathogenies, including chronic inflammation and imbalanced autophagy level, and targeting the associations of circadian rhythm and autophagy is promising strategies in the management and treatment of COPD. Herein, we reviewed the mechanisms of the circadian clock and the unbalance of the autophagic level in COPD, as well as the link between the two, so as to provide further theoretical bases for the study on the pathogenesis of COPD.
\end{abstract}

\section{Introduction}

The 2021 GOLD Guideline classifies COPD as one of the top three causes of death worldwide [1]. The injury of the alveolar epithelium and inflammatory stimulation induced by repeated cigarette smoke (CS) exposure are key pathological processes in the onset and development of COPD, while CS-induced circadian clock dysfunction in COPD may promote the progression of COPD through inflammatory responses and oxidative stress [2]. Hwang et al. found that CS-stimulated mice have significantly different lung functions during day and night [3], which may be related to rhythm changes of CS-mediated surfactant proteins and pulmonary inflammation [4]. Therefore, inflammatory changes induced by pulmonary clock dysfunction may explain COPD's aggravation in poor functioning lungs during nighttime and early morning. Meanwhile, autophagy, above or below the baseline, has been shown to be related to cellular inflammatory responses. Overactivated autophagy can induce allergic airway inflammation in a murine model of respiratory diseases by activating oxidative stress [5]. Conversely, the loss of autophagy-related proteins (e.g., Beclin1, LC3B, ATG5, ATG 4b, and ATG 7) exacerbate neutrophilic infiltration, activate inflammasome (e.g., CXCL1 and NLRP3), and facilitate significant alterations in proinflammatory cytokines $[6,7]$. These observations prove that COPD involves circadian clock dysfunction and autophagy dysregulation, thus suggesting it is necessary to investigate the relationship between circadian clock and autophagy.

\section{The Role of the Circadian Clock in the Regulation of COPD}

The brain's suprachiasmatic nucleus (SCN) receives electrical signals from the retina following light stimulation. Simultaneously, the effect of this photic information is synchronized to the oscillation of central clocks and transmits 


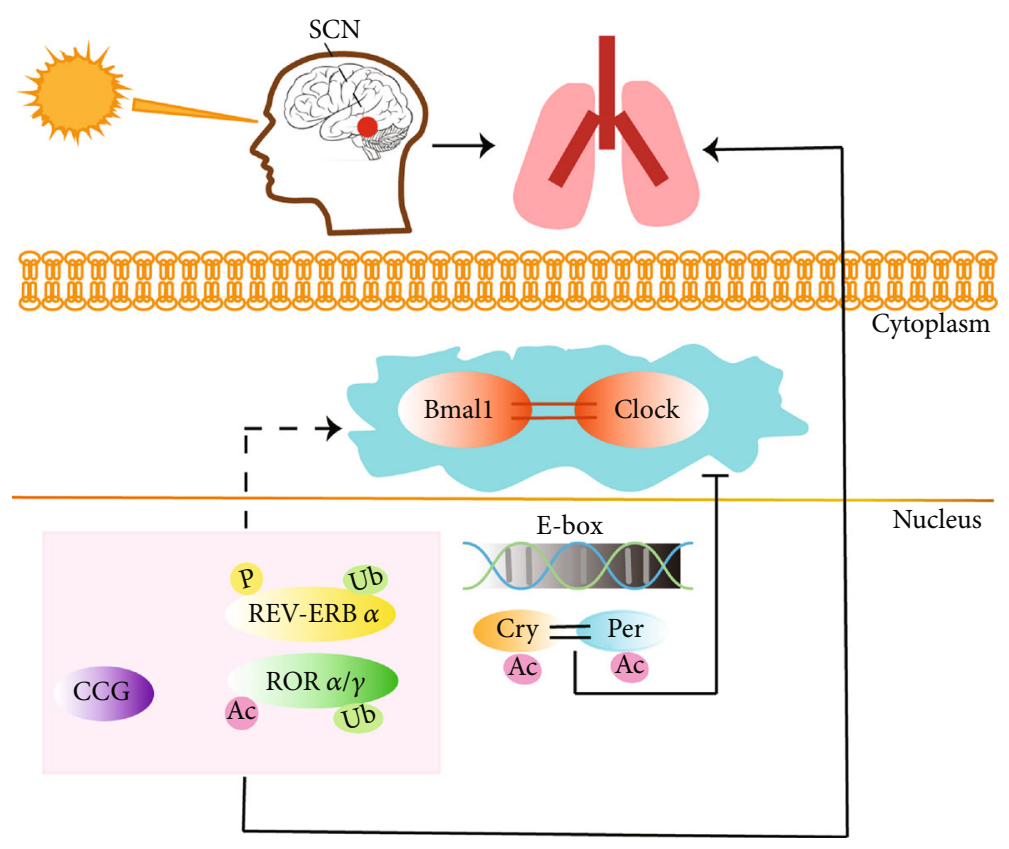

FIgURE 1: The regulation of circadian rhythm in the lung. The peripheral clock present in the lung is affected by SCN. In the form of a heterodimer, the clock/Bmall complex can activate the element sequence of E-box and regulate the expression of the Period family and Cryptochrome family. Per and Cry heterodimers are transmitted to the nucleus after being phosphorylated by casein kinases. They inhibit Per and Cry transcriptions by blocking the activity of the clock/Bmall complex. Nuclear receptors REV-ERB $\alpha / \beta, \mathrm{ROR} \alpha / \gamma$, and CCG, are also involved in the expression of the circadian rhythm of their target genes by regulating transcription. CK: casein kinases; P: phosphorylate; Ub: ubiquitination; Ac: acetylation.

rhythmic information of SCN to peripheral organs and clocks [8]. Its operation is based on the feedback loop of the "transcription-translation" mechanism [9]. As the core factors of circadian rhythm, Bmall and clock have a crucial role in activating the feedback loop [10]. Heterodimers composed of Bmall and clock activate the element sequence of E-box and then regulate the transcription of genes [11]. By binding and activating in the E-box region, the complex of Bmall and clock (Bmall/clock complex) regulates the expression of the Period (Per 1, 2, and 3) family and Cryptochrome (Cry 1 and 2) family. After the translation, the Per and Cry heterodimers are phosphorylated by casein kinases and are transmitted to the nucleus, where they inhibit Per and Cry transcription by blocking the activity of the Bmal1/clock complex [12]. In addition to Per/Cry, nuclear receptors $\mathrm{REV}-\mathrm{ERB} \alpha / \beta$ and $\mathrm{ROR} \alpha / \gamma$ are also involved in the expression of the circadian rhythm of their target genes by regulating transcription [13]. Besides, the clock proteins are observably affected by posttranslational modifications. The modifications of posttranslation regulate protein renewal, intracellular localization, and DNA binding affinity and change the clock proteins' activity and stability through a series of circadian processes [14-16]. Meanwhile, the clockcontrolled genes (CCG) are also affected by a molecular oscillator [12]. The abnormalities in clock gene function interfere with the timing and amplitude of CCG and the circadian processes they control and may lead to chronic diseases like asthma and Alzheimer's disease [16-19] (Figure 1).

Dysfunction of the circadian clock may promote COPD progression via inflammatory responses and oxidative stress [20]. Dysfunction of the circadian clock has been extensively studied in patients or experimental animals with lung diseases. In the lungs of mice with chronic CS exposure, the expression of Bmal1, REV-ERB $\alpha / \beta$ (Nr1d1), and Per1 decrease, and the express process of Per1 and Per2 may also change. Moreover, clinical studies have found a decrease in the number of Bmal1 in COPD patients' lungs. It is believed that such dysfunction partially results from clock's transcription regulated by acetylated and degraded Bmall and Per2 [3, 21]. Furthermore, the timing and amplitude of the circadian clock gene expression and the assumed impact on CCG expression are changed in rodents exposed to CS and proinflammatory mediators (e.g., LPS and TNF- $\alpha$ ) [22]. According to the report of Yao et al. [21], the central and peripheral circadian clocks are involved in the bronchial epithelial cells (BECs) of mice and humans and the neutrophils of mice's bronchoalveolar lavage fluid. Consistently, these studies also demonstrated that the circadian clock could be modified by inflammation and regulate the timing of inflammatory responses. For example, the clock protein could bind to the nuclear transcription factor $\kappa \mathrm{B}$ (NF$\kappa \mathrm{B})$ and regulate its transcription activity [23]. These results suggest damaged oscillating timing, strength, and amplitude of circadian rhythm molecules in COPD that are accompanied by severer inflammation and oxidative stress.

\section{Imbalanced Autophagy Level in COPD}

Autophagy of physiological baseline can promote energy metabolism, stabilize the intracellular environment, eliminate pathogens, degrade damaged organelles, regulate inflammatory responses, and protect lung tissues [24-26]. For instance, the activation of autophagy-mediated by 
Wnt5A (one of the secreted Wnt glycoprotein ligands) signaling promotes the elimination of bacteria (Pseudomonas aeruginosa and Streptococcus pneumoniae) in macrophages [27]. According to a recent study, epithelial autophagy activated by AMPK has been observed in the lungs of developing mice, while the inhibition of AMPK-mediated autophagy led to reduced lung branching in vitro [28]. Importantly, both deficient and overactivated autophagy has been associated with the progression of COPD.

Long-lasting and repeated CS exposure results in impaired autophagy flux, possibly and partially due to the activation of the SIRT6-IGF-Akt-mTOR signaling pathway $[2,29]$. CS exposure increases the expression of p62, the marker of impaired autophagy, and causes ubiquitinated proteins and the autophagy transcription factor TFEB to aggregate in insoluble protein fractions (aggresome bodies) [30]. These findings reveal the potential mechanism of impaired autophagy [31]. Furthermore, when the autophagy in BECs and lung homogenate of COPD patients are inhibited by 3MA (an autophagy chelation-specific inhibitor), cellular senescence and the secretion of inflammatory mediator interleukin-8 (IL-8) increases [29, 32].

Conversely, acute CS exposure triggers an increase in autophagic flux, which is accompanied by severer inflammation, and cellular senescence [30]. In BECs of mice, the expressions of LC3-II, Atg4B, Atg5-Atg12, and Atg7 significantly increased, autophagy vesicles were formed, and inflammatory cytokines (including TNF- $\alpha$, IL-6, and IL-8) were upregulated $[33,34]$. Takasaka et al. found that CS exposure promotes the autophagy activation of human bronchial epithelial cells (HBECs) and accelerates their aging by mediating the histone deacetylase SIRT6 [35]. CS induces autophagy by inhibiting mTOR (a negative regulator of autophagy) in epithelial cells of mice and humans. Mice with a knockout of Mtor in the bronchial or alveolar epithelial cells exhibited aggravated airway inflammation and enlarged airspace [36].

\section{The Link between Clock Molecules and Autophagy in COPD}

The regulatory relationship between autophagy and circadian rhythm molecules was first discovered in the 1970s. Scientists found a diurnal rhythm of autophagic vacuolization and liver atrophy in meal-fed rats [37] and a circadian rhythm of autophagic core factors in drosophila, zebrafishes, and mammals [38-40]. With the development of molecular biology, the mechanism of action between autophagy and circadian rhythm molecules was gradually revealed. Subsequent studies have shown that the autophagy level in different tissues of different species can be controlled by multiple clock genes [39, 41, 42], especially Per2, Bmal1, and clock, which correlate with autophagy. For example, Per2, as the scaffolding protein in the liver, inhibits the activity of mTORC1 via recruiting the tuberous sclerosis complex 1 (TSC1) to mTORC1 complex [43]. In vitro experiments showed that the transient overexpression of Per2 led to the downregulation of the PI3K Class1/Akt pathway and an elevation in the autophagy flux $[44,45]$. In addition, clock mutants reduced the degradation of Bmall through the autophagic pathway via proteasomes and under high-fat feeding, indicating that core clock components are involved in autophagy regulation [46] (Figure 2).

The interrelation between clock molecules and autophagy in lung tissues has been demonstrated in another study. As a histone deacetylase, SIRT1 has been proved to be able to activate autophagy. Lower activity of SIRT1 in the lung epithelial cells and macrophages of humans and mice exposed to CS further induces highly acetylated Bmal1, Per2, and clock proteins or clock gene-related histones and thus promotes inflammation and cell aging [50,51]. All such results prove that both circadian clock dysfunction and imbalanced autophagy can contribute to COPD's development by promoting inflammatory responses, oxidative stress, or cellular senescence.

4.1. REV-ERB $\alpha$ and TFEB/TFE3 Participate in the Rhythmic Expression of Autophagy. REV-ERB $\alpha$ (Nr1d1) is an important component in core circadian clock molecules. Its expression follows the autophagy inhibition pattern basing on the circadian rhythm [52] and relates autophagy to the circadian clock. According to the research of Pariollaud et al., the expression of the REV-ERB $\alpha$ protein in the lung follows the diurnal variation. When REV-ERB $\alpha$ is knocked out, the rhythmic diurnal variation disappears, while inflammatory cytokines significantly increase in the lung of mice [53]. These data indicate that circadian clock molecules may mediate REV-ERB $\alpha$ to promote inflammatory responses and exacerbating COPD's progression. Furthermore, TFEB and TFE3, which are the key regulatory factors for autophagy, lysosomal biogenesis, and cellular homeostasis with circadian rhythm, regulate circadian clock genes involved in autophagy and the expression of REV-ERB $\alpha$ (Nr1d1) [54]. Enrichment of site-binding TFE3 and REV$\mathrm{ERB} \alpha$ is observed in promoter regions of genes (e.g., LAMP1, Mcoln1, Vps33a, Atg3, ATG5, CTSL, and Gabarapl1) involved in the circadian rhythm, autophagy, and lysosomal biogenesis. Meanwhile, the ChIP analysis from Pastore et al. showed several ChIP-seq peaks of TFEB or TFE3 in the promoter of REV-ERB $\alpha$ [54]. Inflammationoxidative stress promoted by autophagy impairment mediated by perinuclear aggregation of TFEB is one of the significant pathological features of CS-induced COPDemphysema [31]. This evidence suggests that TFEB or TFE3 (TFEB/TFE3) can bind to REV-ERB $\alpha$ to participate in autophagy's rhythmic expression and thus regulate pulmonary homeostasis [55].

4.2. $C / E B P \beta$ Integrates the Rhythmic Expression of Autophagy Genes. As a nuclear transcription factor, C/EBP $\beta$ participates in coordinating the rhythmic expression of autophagy genes. In physiological regulation, C/EBP $\beta$ integrates the signals from the circadian rhythm to autophagy. For example, Ma et al. proved the rhythmic induction of $\mathrm{C} / \mathrm{EBP} \beta$ in autophagy genes (e.g., Ulk1, Gabarapl1, LC3B, and Bnip3) in the liver and found that autophagy was related to the circadian clock and maintained the dynamic balance of nutrients throughout the light/dark cycle [55]. In 


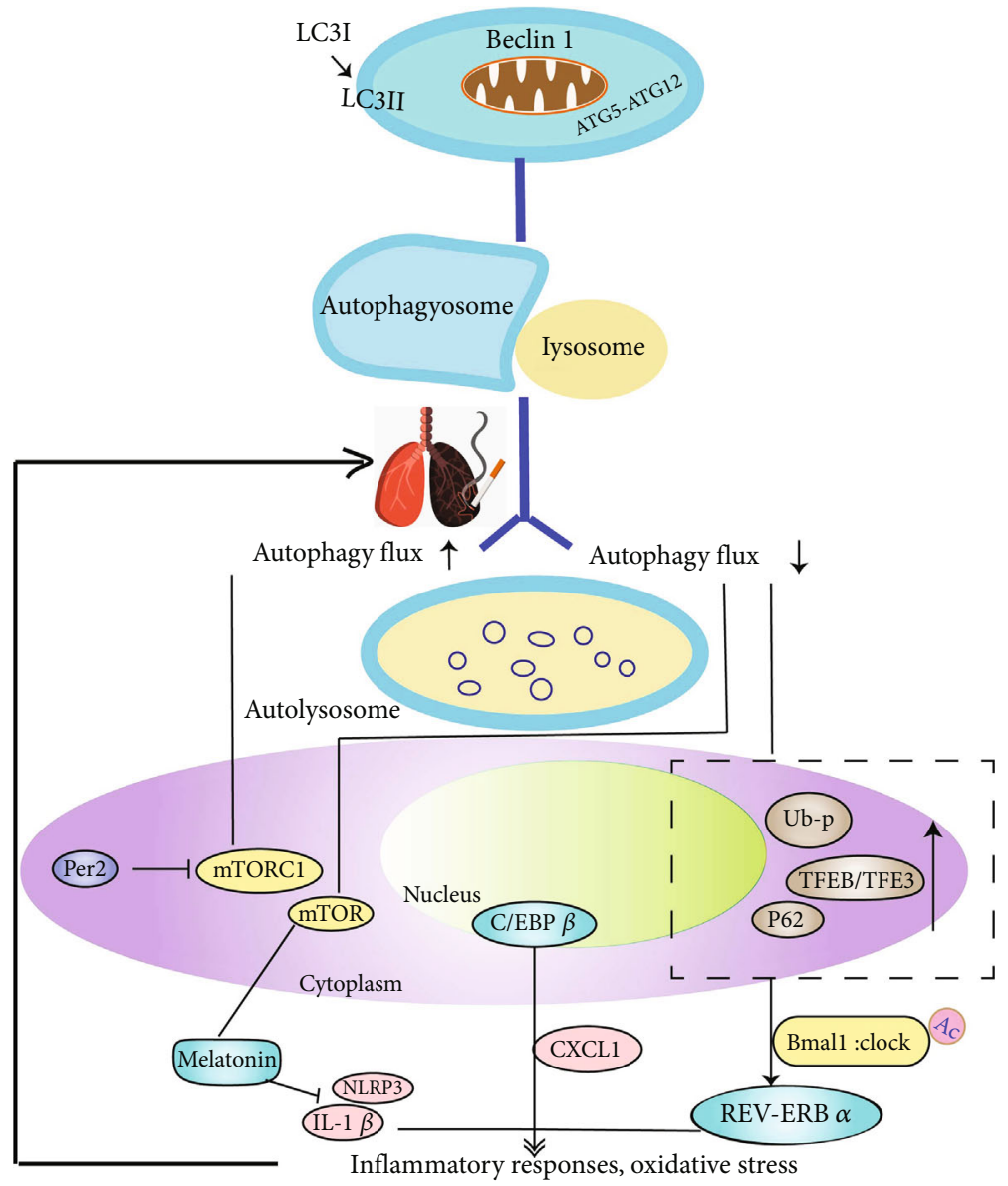

FIGURE 2: Circadian rhythm and autophagy regulation in COPD. The conversion of LC3-I to LC3-II is regarded as an indicator of autophagosome formation [47]. Meanwhile, autophagy proteins BECLIN 1 and ATG5-ATG12 are also involved in the formation of autophagosomes [48]. Autophagosome and lysosome form autolysosome under the digestion of lysosomal hydrolase [49]. CS exposure breaks the balance of the autophagy level and promotes COPD progression by enhancing inflammation and oxidative stress. The core clock protein Per2 can activate autophagy by specifically inhibiting the activity of mTORC1. mTOR in the macromolecular complex (mTOR complex 1 [(mTORC1)) negatively regulates autophagy and activates melatonin, while melatonin inhibits the NLRP3 inflammasome and IL- $1 \beta$ to alleviate airway inflammation. C/EBP $\beta$ is involved in COPD development by participating in the expression of the inflammatory mediator CXCL1 in HBECs and the aggregation of neutrophils. CS exposure increases the expression of p62, inducing aggregation of ubiquitinated proteins and the TFEB in aggresome-bodies while participate in autophagy by regulating core clock genes. p62: the autophagy impairment marker; TFEB: autophagy transcription factor; REV-ERB $\alpha$ (Nr1d1): an important component in core circadian clock molecules; Ub-p, ubiquitinated protein.

pathological regulation, $\mathrm{C} / \mathrm{EBP} \beta$, which has a notable relation with diurnal rhythm, has been proved to be involved in COPD regulation by participating in the expression of the inflammatory mediator CXCL1 in HBECs and the aggregation of neutrophils [56].

4.3. Melatonin Can Inhibit Autophagy by Acting against Inflammation and Antioxidants. As an endocrine hormone, melatonin has been synthesized and secreted by the pineal gland in the brain. It participates in maintaining the normal operation of the circadian clock and regulating autophagy precursors to significantly enhance its protective effect on different systems, including the central nervous system, endocrine system, cardiovascular system, gastrointestinal system, and respiratory system [57-59]. It has been defined that melatonin strongly acts against inflammation and oxidation $[60,61]$. Autophagy is believed to be an induced response to oxidative stress [7]. Accordingly, the antioxidant activity of melatonin can be explained as its inhibition against autophagy [62], which appears to be realized through mTOR activation and the JNK/Bcl-2/Beclin1 signaling pathway [63, 64]. Melatonin is known to inhibit ciclosporin-induced autophagy in rat pituitary GH3 cells through the MAPK/ERK pathway, which is completely or partially attributed to its antioxidant property [58]. Specifically, in rats with COPD, melatonin can inhibit the NLRP3 inflammasome and IL- $1 \beta$, thus alleviating airway inflammation [62], suggesting that melatonin can delay the onset and development of COPD by acting against inflammation.

4.4. Circadian Clock Molecules Regulate mTORC1 in Two Ways. mTOR, a negative regulator of autophagy, is potentially involved in the inflammatory responses of pulmonary diseases. When the expression of mTOR is downregulated, 
the inflammation of COPD is activated, resulting in emphysema [65]. Existing studies have proved that the mTOR can be regulated by the circadian clock genes in two ways: (1) the core clock protein Per2 can inhibit the activity of mTORC1 from activating autophagy [39] and (2) circadian clockrelated transcription factors positively regulate mTORC1. For instance, REV-ERB $\alpha$ can activate the mTORC1 pathway to inhibit autophagy and phosphorylation of the clock protein Bmal1 [66], and similarly, TFEB can activate mTORC1 by inducing cellular endocytosis and formation the endosomes carrying lysosomes [67].

\section{Conclusion and Perspectives}

Few studies have suggested that both autophagy and the circadian clock play an important role in COPD's pathogenesis. However, existing reports have provided possible insights and clues for the interaction between the two. Inflammatory responses and oxidative stress in COPD are the most common factors that lead to imbalanced autophagy and circadian rhythm dysfunction. They are also the main pathological cause of imbalanced autophagy and circadian rhythm dysfunction involved in COPD progression. In this review, we have discussed a possible link between the circadian clock and autophagy via intermediates, such as REV$\mathrm{ERB} \alpha, \mathrm{C} / \mathrm{EBP} \beta, \mathrm{mTORC} 1$, and melatonin, which may all promote COPD progression by inducing inflammatory responses. The specific mechanism of the interrelation between the circadian clock and autophagy in COPD will be further investigated in future studies to provide theoretical bases for the study on COPD's pathogenesis.

\section{Conflicts of Interest}

The authors declare no conflict of interest.

\section{Authors' Contributions}

Writing-original draft preparation and writing-review and editing were done by Y.D.H. Writing-original draft preparation and graphics were done by T.T.H. Conceptualization, supervision, and writing-review and revising were done by J.Z., J.C.D., and Z.G.L.; visualization, X.L.W., J.B.T. All authors have read and agreed to the published version of the manuscript. Yuedi Hu and Tiantian He are considered the co-first authors. Jie Zhu and Jingcheng Dong are considered the cocorrespondence authors.

\section{Acknowledgments}

This study is supported by the National Natural Science Foundation of China (grant number 81974569), China Postdoctoral Science Foundation (grant number 2018M640345), Key Program of Anhui University Natural Science Research (grant number KJ2018A0277), and National Natural Science Foundation of China, Joint Fund for Regional Innovation and Development (grant number U20A20398).

\section{References}

[1] "Global strategy for the diagnosis, management and prevention of COPD," 2021, http://goldcopd.org/2020-gold-globalreports/.

[2] X.-X. Lv, S.-S. Liu, K. Li, B. Cui, C. Liu, and Z.-W. Hu, "Cigarette smoke promotes COPD by activating plateletactivating factor receptor and inducing neutrophil autophagic death in mice," Oncotarget, vol. 8, no. 43, pp. 74720-74735, 2017.

[3] J. Hwang, I. K. Sundar, H. Yao, M. T. Sellix, and I. Rahman, "Circadian clock function is disrupted by environmental tobacco/cigarette smoke, leading to lung inflammation and injury via a SIRT1-BMAL1 pathway," The FASEB Journal, vol. 28, no. 1, pp. 176-194, 2014.

[4] I. K. Sundar, S. Chung, J. Hwang et al., "Mitogen- and stressactivated kinase 1 (MSK1) regulates cigarette smoke-induced histone modifications on NF-KB-dependent genes," PLoS One, vol. 7, no. 2, article e31378, 2012.

[5] S. Gong, X. Ji, J. Su et al., "Yeast fermentate prebiotic ameliorates allergic asthma, associating with inhibiting inflammation and reducing oxidative stress level through suppressing autophagy," Mediators of Inflammation, 13 pages, 2021.

[6] A. C. Racanelli, S. A. Kikkers, A. M. K. Choi, and S. M. Cloonan, "Autophagy and inflammation in chronic respiratory disease," Autophagy, vol. 14, no. 2, pp. 221-232, 2018.

[7] K. Nakahira, S. M. Cloonan, K. Mizumura, A. M. K. Choi, and S. W. Ryter, "Autophagy: a crucial moderator of redox balance, inflammation, and apoptosis in lung disease," Antioxidants \& Redox Signaling, vol. 20, no. 3, pp. 474-494, 2014.

[8] C. Dibner, U. Schibler, and U. Albrecht, "The mammalian circadian timing system: organization and coordination of central and peripheral clocks," Annual Review of Physiology, vol. 72, no. 1, pp. 517-549, 2010.

[9] C. Scheiermann, J. Gibbs, L. Ince, and A. Loudon, "Clocking in to immunity," Nature Reviews. Immunology, vol. 18, no. 7, pp. 423-437, 2018.

[10] S. Sahar and P. Sassone-Corsi, "The epigenetic language of circadian clocks. In Circadian Clocks," in Handbook of Experimental Pharmacology, A. Kramer and M. Merrow, Eds., vol. 217, pp. 29-44, Springer Berlin Heidelberg, Berlin, Heidelberg, 2013.

[11] R. V. Kondratov, "BMAL1-dependent circadian oscillation of nuclear CLOCK: posttranslational events induced by dimerization of transcriptional activators of the mammalian clock system," Genes \& Development, vol. 17, no. 15, pp. 19211932, 2003.

[12] I. Robinson and A. B. Reddy, "Molecular mechanisms of the circadian clockwork in mammals," FEBS Letters, vol. 588, no. 15, pp. 2477-2483, 2014.

[13] A. B. Reddy and G. Rey, "Metabolic and nontranscriptional circadian clocks: eukaryotes," Annual Review of Biochemistry, vol. 83, no. 1, pp. 165-189, 2014.

[14] J. A. Evans and A. J. Davidson, "Health consequences of circadian disruption in humans and animal models," in In Progress in Molecular Biology and Translational Science, vol. 119, pp. 283-323, Elsevier, Amsterdam, 2013.

[15] Y. Nakahata, M. Kaluzova, B. Grimaldi et al., "The NAD+dependent deacetylase SIRT1 modulates CLOCK-mediated chromatin remodeling and circadian control," Cell, vol. 134, no. 2, pp. 329-340, 2008. 
[16] J. Hirayama, S. Sahar, B. Grimaldi et al., "CLOCK-mediated acetylation of BMAL1 controls circadian function," Nature, vol. 450, no. 7172, pp. 1086-1090, 2007.

[17] Circadian Clocks and A. Kramer, Handbook of Experimental Pharmacology, M. Merrow, Ed., vol. 217, Springer Berlin Heidelberg, Berlin, Heidelberg, 2013.

[18] L. Y. Fang, Y. Y. Liu, and J. W. Guan, "Research progress on the relationship between Alzheimer's disease and biological rhythm," Chinese Journal of Gerontology, vol. 31, no. 8, pp. 1486-1488, 2011.

[19] S. Gery and H. P. Koeffler, "Circadian rhythms and cancer," Cell Cycle, vol. 9, no. 6, pp. 1097-1103, 2010.

[20] I. K. Sundar, H. Yao, M. T. Sellix, and I. Rahman, "Circadian molecular clock in lung pathophysiology," American Journal of Physiology-Lung Cellular and Molecular Physiology, vol. 309, no. 10, pp. L1056-L1075, 2015.

[21] H. Yao, I. K. Sundar, Y. Huang et al., "Disruption of sirtuin 1mediated control of circadian molecular clock and inflammation in chronic obstructive pulmonary disease," American Journal of Respiratory Cell and Molecular Biology, vol. 53, no. 6, pp. 782-792, 2015.

[22] S. Gebel, B. Gerstmayer, P. Kuhl, J. Borlak, K. Meurrens, and T. Müller, "The kinetics of transcriptomic changes induced by cigarette smoke in rat lungs reveals a specific program of defense, inflammation, and circadian clock gene expression," Toxicological Sciences, vol. 93, no. 2, pp. 422-431, 2006.

[23] I. K. Sundar, M. T. Sellix, and I. Rahman, "Redox regulation of circadian molecular clock in chronic airway diseases," Free Radical Biology \& Medicine, vol. 119, pp. 121-128, 2018.

[24] S. Jiang, J. Sun, N. Mohammadtursun et al., "Dual role of autophagy/mitophagy in chronic obstructive pulmonary disease," Pulmonary Pharmacology \& Therapeutics, vol. 56, pp. 116-125, 2019.

[25] K. Nakahira, M. A. Pabon Porras, and A. M. K. Choi, “Autophagy in pulmonary diseases," American Journal of Respiratory and Critical Care Medicine, vol. 194, no. 10, pp. 1196-1207, 2016.

[26] S. Jati, S. Kundu, A. Chakraborty, S. K. Mahata, V. Nizet, and M. Sen, "Wnt 5A signaling promotes defense against bacterial pathogens by activating a host autophagy circuit," Frontiers in Immunology, vol. 9, p. 679, 2018.

[27] B. Yeganeh, J. Lee, L. Ermini, I. Lok, C. Ackerley, and M. Post, "Autophagy is required for lung development and morphogenesis," The Journal of Clinical Investigation, vol. 129, no. 7, pp. 2904-2919, 2019.

[28] S. Aggarwal, P. Mannam, and J. Zhang, "Differential regulation of autophagy and mitophagy in pulmonary diseases," American Journal of Physiology-Lung Cellular and Molecular Physiology, vol. 311, no. 2, pp. L433-L452, 2016.

[29] S. Fujii, H. Hara, J. Araya et al., "Insufficient autophagy promotes bronchial epithelial cell senescence in chronic obstructive pulmonary disease," Onco Immunology, vol. 1, no. 5, pp. 630-641, 2012.

[30] M. Bodas, C. V. Westphal, R. Carpenter-Thompson, D. K. Mohanty, and N. Vij, "Nicotine exposure induces bronchial epithelial cell apoptosis and senescence via ROS mediated autophagy-impairment," Free Radical Biology and Medicine, vol. 97, pp. 441-453, 2016.

[31] M. Bodas, N. Patel, D. Silverberg, K. Walworth, and N. Vij, "Master autophagy regulator transcription factor EB regulates cigarette smoke-induced autophagy impairment and chronic obstructive pulmonary disease-emphysema pathogenesis," Antioxidants \& Redox Signaling, vol. 27, no. 3, pp. 150-167, 2017.

[32] Y. Tang, Q.-H. Cai, Y.-J. Wang et al., "Protective effect of autophagy on endoplasmic reticulum stress induced apoptosis of alveolar epithelial cells in rat models of COPD," Bioscience Reports, vol. 37, no. 6, 2017.

[33] S. W. Ryter and A. M. K. Choi, "Autophagy in lung disease pathogenesis and therapeutics," Redox Biology, vol. 4, pp. 215-225, 2015.

[34] K. Wang, Y. Chen, P. Zhang, P. Lin, N. Xie, and M. Wu, "Protective features of autophagy in pulmonary infection and inflammatory diseases," Cell, vol. 8, no. 2, p. 123, 2019.

[35] N. Takasaka, J. Araya, H. Hara et al., "Autophagy induction by SIRT6 through attenuation of insulin-like growth factor signaling is involved in the regulation of human bronchial epithelial cell senescence," The Journal of Immunology, vol. 192, no. 3, pp. 958-968, 2014.

[36] Y. Wang, J. Liu, J.-S. Zhou et al., "MTOR suppresses cigarette smoke-induced epithelial cell death and airway inflammation in chronic obstructive pulmonary disease," Journal of Immunology, vol. 200, no. 8, pp. 2571-2580, 2018.

[37] U. Pfeifer, "Cellular autophagy and cell atrophy in the rat liver during long-term starvation," Virchows Archiv B Cell Pathology, vol. 12, pp. 195-211, 1972.

[38] D. Ma, S. Panda, and J. D. Lin, “Temporal orchestration of circadian autophagy rhythm by $\mathrm{C} / \mathrm{EBP} \beta$ : $\mathrm{C} / \mathrm{EBP} \beta$ regulates circadian autophagy rhythm," The EMBO Journal, vol. 30 , no. 22, pp. 4642-4651, 2011.

[39] E. Kijak and E. Pyza, “TOR signaling pathway and autophagy are involved in the regulation of circadian rhythms in behavior and plasticity of L2 interneurons in the brain of drosophila melanogaster," PLoS One, vol. 12, no. 2, article e0171848, 2017.

[40] G. Huang, F. Zhang, Q. Ye, and H. Wang, "The circadian clock regulates autophagy directly through the nuclear hormone receptor $\mathrm{Nr} 1 \mathrm{~d} 1 / \mathrm{Rev}-\mathrm{Erb} \alpha$ and indirectly via $\mathrm{Cebpb} /(\mathrm{C} / \mathrm{Ebp} \beta)$ in zebrafish," Autophagy, vol. 12, no. 8, pp. 1292-1309, 2016.

[41] X. Chen, K. Kondo, K. Motoki, H. Homma, and H. Okazawa, "Fasting activates macroautophagy in neurons of Alzheimer's disease mouse model but is insufficient to degrade amyloidbeta," Scientific Reports, vol. 5, no. 1, p. 12115, 2015.

[42] U. Pfeifer and H. Scheller, "A morphometric study of cellular autophagy including diurnal variations in kidney tubules of normal rats," The Journal of Cell Biology, vol. 64, no. 3, pp. 608-621, 1975.

[43] R. Wu, F. Dang, P. Li et al., "The circadian protein period 2 suppresses MTORC1 activity via recruiting Tsc1 to MTORC1 complex,” Cell Metabolism, vol. 29, no. 3, pp. 653-667e6, 2019.

[44] F. Kalfalah, L. Janke, A. Schiavi et al., "Crosstalk of clock gene expression and autophagy in aging," Aging, vol. 8, no. 9, pp. 1876-1895, 2016.

[45] Z. Wang, L. Li, and Y. Wang, "Effects of Per2 overexpression on growth inhibition and metastasis, and on MTA1, Nm23$\mathrm{H} 1$ and the autophagy-associated PI3K/PKB signaling pathway in nude mice xenograft models of ovarian cancer," Molecular Medicine Reports, vol. 13, no. 6, pp. 4561-4568, 2016.

[46] K. Jeong, B. He, K. Nohara et al., "Dual attenuation of proteasomal and autophagic BMAL1 degradation in Clock $\Delta 19 /+$ mice contributes to improved glucose homeostasis," Scientific Reports, vol. 5, no. 1, p. 12801, 2015. 
[47] N. Mizushima, T. Yoshimori, and B. Levine, "Methods in mammalian autophagy research," Cell, vol. 140, no. 3, pp. 313-326, 2010.

[48] B. Ravikumar, S. Sarkar, J. E. Davies et al., "Regulation of mammalian autophagy in physiology and pathophysiology," Physiological Reviews, vol. 90, no. 4, pp. 1383-1435, 2010.

[49] N. Mizushima and M. Komatsu, "Autophagy: renovation of cells and tissues," Cell, vol. 147, no. 4, pp. 728-741, 2011.

[50] I. K. Sundar, H. Yao, M. T. Sellix, and I. Rahman, "Circadian clock-coupled lung cellular and molecular functions in chronic airway diseases," American Journal of Respiratory Cell and Molecular Biology, vol. 53, no. 3, pp. 285-290, 2015.

[51] I. Tran, C. Ji, I. Ni, T. Min, D. Tang, and N. Vij, "Role of cigarette smoke-induced aggresome formation in chronic obstructive pulmonary disease-emphysema pathogenesis," American Journal of Respiratory Cell and Molecular Biology, vol. 53, no. 2, pp. 159-173, 2015.

[52] B. Grimaldi, "Lysosomotropic REV-ERB antagonism: a metabolic connection between circadian rhythm and autophagy may tell cancer cells it's time to die," Molecular \& Cellular Oncology, vol. 2, no. 2, article e965626, 2015.

[53] M. Pariollaud, J. E. Gibbs, T. W. Hopwood et al., "Circadian clock component REV-ERB $\alpha$ controls homeostatic regulation of pulmonary inflammation," Journal of Clinical Investigation, vol. 128, no. 6, pp. 2281-2296, 2018.

[54] N. Pastore, A. Vainshtein, N. J. Herz et al., "Nutrient-sensitive transcription factors TFEB and TFE 3 couple autophagy and metabolism to the peripheral clock," The EMBO Journal, vol. 38, no. 12, 2019.

[55] D. Ma, S. Panda, and J. D Lin, “Temporal orchestration of circadian autophagy rhythm by C/EBPß: C/EBPß regulates circadian autophagy rhythm," The EMBO Journal, vol. 30, no. 22, pp. 4642-4651, 2011.

[56] A. B. Roos, J. L. Barton, A. Miller-Larsson et al., "Lung epithelial-C/EBP $\beta$ contributes to LPS-induced inflammation and its suppression by formoterol," Biochemical and Biophysical Research Communications, vol. 423, no. 1, pp. 134-139, 2012.

[57] A. Roohbakhsh, A. Shamsizadeh, A. W. Hayes, R. J. Reiter, and G. Karimi, "Melatonin as an endogenous regulator of diseases: the role of autophagy," Pharmacological Research, vol. 133, pp. 265-276, 2018.

[58] V. Motilva, S. García-Mauriño, E. Talero, and M. Illanes, "New paradigms in chronic intestinal inflammation and colon cancer: role of melatonin: intestinal inflammation, colon cancer, and melatonin," Journal of Pineal Research, vol. 51, no. 1, pp. 44-60, 2011.

[59] W. Wang and J. Gao, "Effects of melatonin on protecting against lung injury (review)," Experimental and Therapeutic Medicine, vol. 21, no. 3, p. 228, 2021.

[60] R. Hardeland, "Melatonin and inflammation-story of a double-edged blade," Journal of Pineal Research, vol. 65, no. 4, article e12525, 2018.

[61] R. J. Reiter, S. Rosales-Corral, D. X. Tan, M. J. Jou, A. Galano, and B. Xu, "Melatonin as a mitochondria-targeted antioxidant: one of evolution's best ideas," Cellular and Molecular Life Sciences, vol. 74, no. 21, pp. 3863-3881, 2017.

[62] Z. Peng, W. Zhang, J. Qiao, and B. He, "Melatonin attenuates airway inflammation via SIRT1 dependent inhibition of NLRP3 inflammasome and IL- $1 \beta$ in rats with COPD," International Immunopharmacology, vol. 62, pp. 23-28, 2018.
[63] P. Kongsuphol, S. Mukda, C. Nopparat, A. Villarroel, and P. Govitrapong, "Melatonin attenuates methamphetamineinduced deactivation of the mammalian target of rapamycin signaling to induce autophagy in SK-N-SH cells," Journal of Pineal Research, vol. 46, no. 2, pp. 199-206, 2009.

[64] C. Nopparat, J. E. Porter, M. Ebadi, and P. Govitrapong, "The mechanism for the neuroprotective effect of melatonin against methamphetamine-induced autophagy: melatonin protects against methamphetamine-induced autophagy," Journal of Pineal Research, vol. 49, no. 4, pp. 382-389, 2010.

[65] I. C. Nnah, B. Wang, C. Saqcena et al., "TFEB-driven endocytosis coordinates MTORC1 signaling and autophagy," Autophagy, vol. 15, no. 1, pp. 151-164, 2019.

[66] M. Dadon-Freiberg, N. Chapnik, and O. Froy, "REV-ERB $\alpha$ alters circadian rhythms by modulating MTOR signaling," Molecular and Cellular Endocrinology, vol. 521, p. 111108, 2021.

[67] T. Yoshida, I. Mett, A. K. Bhunia et al., "Rtp801, a suppressor of MTOR signaling, is an essential mediator of cigarette smoke-induced pulmonary injury and emphysema," Nature Medicine, vol. 16, no. 7, pp. 767-773, 2010. 